\title{
Observations of Comets
}

made at the U. S. N. Observatory, Washingtor, with the 9.6inch Equatorial, by Prof. E. Frisby. (Communicated by the Superintendent, Commodore George E. Belknap, U. S. N:)

\begin{tabular}{|c|c|c|c|c|c|c|c|c|c|c|}
\hline Date & Wash. M.T. & $\Delta \alpha$ & $\Delta \delta$ & Comp. & $\alpha$ app. & $\log p .4$ & $\delta$ app. & $\log p \cdot \Delta$ & Red. ad l. app. & * \\
\hline \multicolumn{11}{|c|}{ Comet $1886 \ldots($ Fabry $)}$. \\
\hline ec. 2 & $9^{\mathrm{h}} 20^{\mathrm{m}} \quad \mathbf{2}^{\mathrm{s}}$ & $-0^{m} 44^{s} \cdot 5^{2}$ & $-9^{\prime} 56.0$ & I 9.4 & $0^{h} 3^{6^{m}+1}: s_{1}$ & 9.285 & $+21^{\circ} 0^{\prime} 20^{\prime \prime} 2$ & 0.460 & $+3 \cdot 5^{s} 8+24^{\prime \prime} \cdot 3$ & $\mathbf{I}$ \\
\hline 3 & $810 \quad 5^{6}$ & 7.09 & -824.8 & 20.4 & ○ $33 \quad 53.93$ & $8.79^{6}$ & $+205^{8} 4^{6.7}$ & 0.433 & $+3.54+24.5$ & 2 \\
\hline 6 & $\begin{array}{lll}6 & 27 & 6\end{array}$ & -125.01 & +921.0 & 20.4 & 0277.81 & $9.079 \mathrm{n}$ & $+2054 \quad 3.5$ & 0.443 & $+3.47+24.7$ & 3 \\
\hline 7 & $6 \quad 5^{8} \quad 54$ & -342.00 & $+753 \cdot 3$ & 20.4 & $\circ 2450.8 \mathrm{I}$ & $8.593 n$ & $+20 \quad 5235.7$ & 0.434 & $+3.46+24.7$ & 3 \\
\hline 11 & 64310 & to $45 \cdot 3 \circ$ & +1025.0 & $20: 4$ & $\begin{array}{lll}\circ & 16 & 24.87\end{array}$ & $8.3^{2} 5 n$ & +204734.9 & 0.435 & $+3 \cdot 32+25 \cdot 4$ & 4 \\
\hline I 2 & $\begin{array}{lll}5 & 43 & 20\end{array}$ & - I 111.17 & +910.4 & 20.4 & o $1428.3^{8}$ & $9.13^{2}$ & +204549.5 & $0.44^{8}$ & $+33^{\circ}+26.0$ & 4 \\
\hline 26 & $\begin{array}{lll}6 & \text { I } 3 & 43\end{array}$ & $-2 \quad 30.35$ & +253.6 & $15 \cdot 3$ & $23 \quad 5^{1} 23.00$ & 8.980 & +204222.9 & 0.444 & $+2.94+26.4$ & 5 \\
\hline 29 & $\begin{array}{lll}6 & 49 & 44\end{array}$ & $4.7^{\circ}$ & - I 244.2 & I $4 \cdot 3$ & $\begin{array}{llll}23 & 47 & 30.65\end{array}$ & 9.304 & +204545.6 & 0.468 & $+2.84+25.4$ & 6 \\
\hline
\end{tabular}

1885

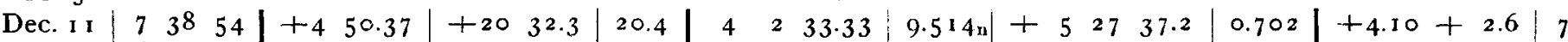

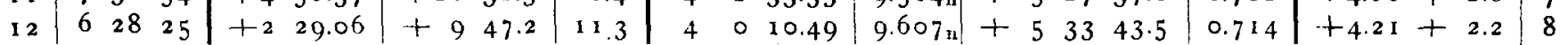

$1885-86$

Dec. $29+6$ Comet $1885 \mathrm{~V}$.

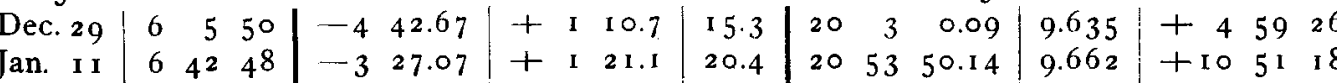

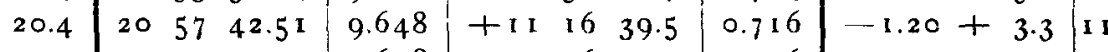

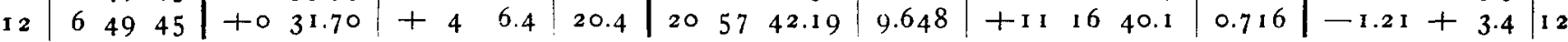
Mean Places of the Comparison Stars.

\begin{tabular}{|c|c|c|c|}
\hline$*$ & $\alpha 1885.0$ & $\delta$ I 885.0 & Authority \\
\hline $\mathbf{I}$ & $\circ^{\mathrm{h}} 3^{6^{\mathrm{m}} 5^{2.5} \circ 5}$ & $+21^{\circ} 9^{\prime} 5^{1 . " 9}$ & $W_{2} \circ^{h} 929-3 \circ$ \\
\hline 2 & - $3^{2} 43 \cdot 3^{\circ}$ & $6 \quad 46.9$ & $W_{2} \circ \mathrm{h} 8 \circ 9-10$ \\
\hline 3 & $\begin{array}{lll}\circ & 28 & 29.35\end{array}$ & +204417.8 & $W_{2} o^{h_{1}} 686-7$ \\
\hline 4 & ○ I 536.25 & $+203^{6}$ I3.1 & $W_{2} o^{h} 367$ \\
\hline 5 & 235350.4 I & $+2039 \quad 2.9$ & $W_{2} 23^{h} 1096$ \\
\hline 6 & $2347 \quad 23.11$ & +2058 & $W_{2} 23^{h} 97^{6}$ \\
\hline
\end{tabular}

Cometenbeobachtungen auf der Sternwarte in Hamburg. Von W. Luther.

\begin{tabular}{|c|c|c|c|c|c|c|c|c|c|c|c|}
\hline г 886 & M.Z.Hamb. & $\Delta \alpha$ & & $\Delta d$ & & $\alpha$ app. & $\log p .4$ & $\delta$ app. & $|\log p \cdot \Delta|$ & Red.ad l. app. & $*$ \\
\hline \multicolumn{12}{|c|}{ Comet $1886 \ldots(\mathrm{Fa}, \mathrm{bry})$} \\
\hline März & $7^{\mathrm{h}} 44^{\mathrm{m}} 10^{\mathrm{s}}$ & $-2^{\mathrm{m}} \quad 6.86$ & - & $0^{\prime} 19.2$ & $23^{h}$ & $19^{\mathrm{m}} 59^{\mathrm{s}} \cdot 57$ & $9 \cdot 586$ & $+30^{\circ} 42^{\prime} \quad 3^{\prime \prime} 8$ & 0.844 & $-0.80-2.1$ & \\
\hline & 73230 & $-0 \quad 31.45$ & & - & 23 & 1943.06 & $9.59 \mathrm{I}$ & - & -- & $-0.79-2.4$ & \\
\hline I & $8 \quad 5 \quad 43$ & $+3 \quad 0.67$ & - & 350.4 & 23 & $19 \quad 14.82$ & 9.555 & +32 & 0.869 & $-0.79-2.8$ & \\
\hline
\end{tabular}

März 5. Luft gut. Sehr heller Kern im Brennpunkt der gleichmässig hellen parabolischen Nebelhülle.

März 7. Nur 2 Vergleichungen. Wolken. - März 10. Mond. Sehr dunstige Luft, Beobachtung unsicher. Comet $1886 \ldots$ (Barnard).

\begin{tabular}{rr|rrr|rr|rrr|lll|l|l|lll|l|l|l} 
März & 5 & 9 & 48 & 30 & +0 & 37.49 & - & 2 & 25.8 & I & 55 & 15.08 & 9.570 & +22 & 16 & 2.8 & 0.843 & -0.40 & -4.4 & 4 \\
I0 & 9 & 18 & 45 & -0 & 20.34 & + & 2 & 22.9 & 1 & 53 & 57.31 & 9.578 & +23 & 42 & 38.4 & 0.835 & -0.45 & -4.6 & 5
\end{tabular}

März 5. Luft gut. - März 10. Schlechte Luft; Mond; später ganz trübe. Mittlere Oerter der Vergleichsterne für $1886,0$.

\begin{tabular}{|c|c|c|c|c|c|c|c|}
\hline$*$ & $\alpha \mathrm{I} 886.0$ & $\delta$ I 886.0 & Autorität & * & $a \mathrm{I} 886.0$ & $\delta$ İ 886.0 & Autorität \\
\hline $\mathbf{I}$ & $23^{\mathrm{h}} 22^{\mathrm{m}} \quad 7^{\mathrm{s}} \cdot 23$ & $+30^{\circ} 42^{\prime} 25^{\prime \prime} 1$ & A. G. Leiden Z. I O I & 4 & $1^{\mathrm{h}} 54^{\mathrm{In}} 37^{\mathrm{s}} \cdot 99$ & $+22^{\circ} 18^{\prime} 33^{\prime \prime} \circ$ & B.Z. 292 \\
\hline $\begin{array}{l}2 \\
3\end{array}$ & $\begin{array}{lll}23 & 20 & 15.30 \\
23 & 16 & 14.94\end{array}$ & $\begin{array}{rrr}+3124 & 6.8 \\
+3212 & 53.2\end{array}$ & $\begin{array}{l}\text { B. Z. } 374 \\
\text { A. G. Leiden Z. } 131\end{array}$ & 5 & I 54 I 8.10 & +234020.1 & B. Z. 445 \\
\hline
\end{tabular}

Inhalt zu Nr. 2717. A. Auwers. Ueber die Declination von 74 Cygni. 65.- M. Wilhelm Meyer. Ueber die Bahn eines unsichtbaren Cometen. 69. - H. Kreutz. Ueber das System der Cometen 1843 I, 1880 I und 1882 II. 73. - W. Zenker. Noch Einiges iiber den Biela'schen Cometen. 75. - Col. Wagner. Cometen-Beobachtungen in Kremsmünster. 77. - George E. Belknap. Observations of Comets. 79. - W. Luther. Cometenbeobachtungen auf der Sternwarte in Hamburg. 79.

Gesehlossen r886 März x8. Herausgeber; A. Krueger. Druck von C. F. Mohr. Expedition: Sternwarte in Kiel. 